Meta

Journal des tradlucteurs

Translators' Journal

\title{
Néologie en marche... arrière ou avant ?
}

\section{Richard Kromp}

Volume 26, numéro 2, juin 1981

URI : https://id.erudit.org/iderudit/003354ar

DOI : https://doi.org/10.7202/003354ar

Aller au sommaire du numéro

Éditeur(s)

Les Presses de l'Université de Montréal

ISSN

0026-0452 (imprimé)

1492-1421 (numérique)

Découvrir la revue

Citer cet article

Kromp, R. (1981). Néologie en marche... arrière ou avant ? Meta, 26(2), 175-182. https://doi.org/10.7202/003354ar d'utilisation que vous pouvez consulter en ligne.

https://apropos.erudit.org/fr/usagers/politique-dutilisation/ 


\section{NÉOLOGIE EN MARCHE... ARRIÈRE OU AVANT ?}

Le téléphone sonne. Je prends mon courage à deux mains et le récepteur... comme je peux. "Terminologie, bonjour?» $\mathrm{Au}$ bout du fil, après quelques excuses sur l'heure qu'il est, et une flatterie ou deux de circonstance, une voix rauque me lance la QUESTION de plein fouet : "Dites-moi rapidement, comment traduisons-nous «multithreading»? J'hésite un moment sur ce que je dois faire, et comme je ne parviens pas à me décider, je dis à mon interlocuteur que je le rappellerai. Bien sûr, la pensée de traduire le «visor» avec lequel une traductrice est aux prises me harcèle toujours.

Les deux problèmes qu'on vient coup sur coup de m'exposer me mettent dans l'embarras. C'est un dilemme qui apparaît chaque fois qu'un problème de néologie se pose. Dans les techniques de pointe, il va de soi que la nécessité de la création néologique se présente souvent. On aurait beau se vouer à tous les diables pour chercher des équivalences consignées, on reviendrait breclouille! Une fois la documentation nécessaire rassemblée, une décision s'impose, décision qu'on voudrait éclairée et certes pas à la lueur d'une... vessie!

Dans le cas qui nous préoccupe, la traductrice cherche l'équivalent français de ce qu'on a appelé "visor» en anglais : il s'agit d'un nouveau dispositif ajouté au plus récent modèle de machine à écrire. Ce dispositif a la forme d'un volet et sert à éviter que l'usager de la machine ne soit ébloui par la lampe qui $y$ est incorporée.

La recherche préliminaire a fourni les renseignements suivants :

- une consultation auprès de la Banque de terminologie d'Ottawa a donné : visor $=$ visière (dans le contexte de l'armée de terre, me dit-on, et puisqu'il s'agit d'une vieille fiche, on n'en sait pas plus long).

- Un coup de téléphone au Dépôt dentaire m'apprend que les petits volets posés en quadrillage autour de la lampe de mon dentiste ne sont pas là pour éviter que la lampe ne m'éblouisse, mais ont pour unique objet de bien éclairer le travail des dentistes (égoïstes !).

- Le Duden parle de dispositif anti-éblouissant (dans le cas de phares d'automobiles).

- Le Petit Robert donne des définitions de volet, de visière et de déflecteur qui ne rendent pas compte de notre nouveau "machin " ${ }^{1}$.

1. Voir annexe $A$. 
Dans le cas de «multithreading", tout ce qu'on connaît, c'est la traduction de «thread» (filière), et les définitions de l'un et de l'autre ${ }^{2}$.

Pour régler ces deux cas, deux procédés principaux s'offrent à nous :

a) recourir à la lexie complexe (appelée aussi composition nominale): par exemple

visor: volet anti-éblouissant dispositif anti-éblouissant

multithreading : traitement multi-filière

b) recourir à la lexie simple

- par extension de sens : visière

- par dérivation : multifiliérisation.

Que faire?

\section{CRITÈRES EN NÉOLOGIE}

Le recours à la lexie complexe, faut-il le souligner, n'est qu'un procédé de création néologique parmi tant d'autres. En effet, selon la typologie établie par P. Auger et L.-J. Rousseau ${ }^{3}$, il y a trois groupes de néologie. D'abord il y a la néologie de forme: la dérivation (en utilisant préfixes ou suffixes), la composition (mots composés ou syntagmes), l'acronymie, les mots-valises (par exemple : chocomousse), la néologie par conversion (changement de catégories grammaticales) et les changements orthographiques (électro-nucléaire $\rightarrow$ électronucléaire). Puis la néologie de sens (par exemple : un cadre supérieur) et enfin, l'emprunt (par exemple : label (en informatique)).

Cependant rien nous assure que chacun de ces procédés soit d'égale valeur. En effet, il existe des critères d'acceptabilité des néologismes qu'Auger et Rousseau distinguent comme suit, sur le plan strictement linguistique :

- l'intégration au système phonologique et orthographique français;

— l'absence de connotations péjoratives ou gênantes (brûlage à la torche $\neq$ torchage) ;

- l'intégration syntaxique, c'est-à-dire la possibilité de dériver, tant sur le plan syntagmatique que sur le plan paradigmatique ou transformationnel;

- le critère onomasiologique ou l'absence de concurrence (relation une notion = un terme);

- la valeur sociolinguistique.

Toujours selon Auger et Rousseau, il faut ajouter aux critères linguistiques, ceux d'un ordre terminologique. Ceux-ci (consensus de différents groupes, sondage des chances d'acceptabilité, décision de comités de référence, emploi par des

2. Voir annexe B.

3. P. Auger et J.-L. Rousseau. Méthodologie de la recherche terminologique, p. 55-57. 
organismes officiels, caution par des comités de normalisation) concernent davantage l'activité de normalisation que la création néologique proprement dite. Mais sans égard au contenu et à la légitimité de l'activité normalisatrice des organismes responsables, il y a lieu de souligner qu'elle ne répond pas aux besoins en néologie propres à notre ère d'innovations et de changements technologiques, sociaux, économiques ou politiques. S'il faut s'en persuader, il suffit de rappeler que R. J. Steiner a évalué à 20 ou 30 ans le retard possible accusé par les dictionnaires pour rendre compte de néologismes, à plus d'un siècle dans le cas des dictionnaires d'Académie ${ }^{4}$. C'est ce qui fait conclure à Steiner :

the work of these commissions (for standardization) has shown little effect upon usage because of the slowness with which the groups work and the small number of words upon which they legislate compared to the great abundance of neologisms resulting from the vast scientific and cultural changes which have taken place ${ }^{5}$.

En effet, on chiffre à 300000 l'augmentation annuelle de composés nouveaux en chimie. Ajoutez à cela les 4,7 millions de composés déjà existants, et multipliez par les quelque 300 à 1000 sous-domaines de l'activité humaine, et vous avez une vague idée de l'ampleur du travail terminologique qu'on peut entreprendre ${ }^{6}$.

On aura beau déplorer cet état de fait, crier au scandale, voire même pleurer sur le sort des pauvres traducteurs aux prises avec ces caméléons à mille têtes, rien ne changera à court terme.

Il faut créer coûte que coûte, sans pouvoir attendre qu'on statue officiellement.

Il faut donc conclure qu'il y a un laps de temps séparant le moment de la création néologique (celui qui nous intéresse) du moment de la normalisation. Par conséquent, les critères terminologiques évoqués par Auger et Rousseau ne sauraient être retenus ici.

Avant de clore la discussion autour des critères à retenir, il y a lieu de mentionner qu'un autre critère semble suspect: le critère onomasiologique. La relation d'unicité terme-notion, si elle est souhaitable, ne respecte pas, semble$\mathrm{t}$-il, le processus normal de formation de nouveaux termes. Il s'agit là cependant d'une idée qui est maintes fois formulée. Nombreux sont les auteurs qui distinguent le terme technique du vocabulaire commun par l'aspect monoréférentiel du premier par rapport à l'aspect polysémique de la langue commune. Il suffit pour s'en persuader, de citer le point de vue de Louis Guilbert :

Le signe société par exemple, qui exprime la relation du concept «société» avec des groupements humains divers, peut prendre un très grand nombre de significations tandis que le signe acier, en tant que signe simple ne

4. Steiner, R. J., Lebende Sprachen, p. 146.

5. Steiner, R. J., op. cit., p. 145.

6. Chiffres avancés par M. Andrejewsky, de Carnegie Mellon, lors d'une conférence donnée à l'U. de M. en février 1980 . 
pourra se référer qu'à un seul matériau. L'un est essentiellement polysémique, l'autre essentiellement monosémique. C'est là que réside la différenciation entre le mot du vocabulaire commun et le terme technique ${ }^{7}$.

Si le critère onomasiologique est important au stade de la normalisation, au moment de la création néologique, il ne correspond pas à un état de fait véritable. Au contraire, il est normal en néologie qu'un certain nombre de solutions intéressantes, particulièrement en milieu de traduction, se côtoient pendant un certain temps, avant que la communauté linguistique ou un organisme de normalisation ne fasse un choix. Voici quelques exemples d'équivalences qui ont existé en concurrence pendant un certain temps :

$\begin{array}{ll}\text { wage-price freeze } & \text { blocage des prix et des salaires } \\ & \text { gel des salaires et des prix } \\ \text { contrôle des prix et des salaires } & \text { gel de tous les prix et salaires } \\ \text { sample home } & \text { maison témoin } \\ \text { model home } & \text { maison exposition } \\ & \text { maison modèle } \\ & \text { villa modèle } \\ & \text { appartement témoin } \\ & \text { appareil de correction auditive } \\ \text { appareil auditif } \\ \text { hearing aid } & \text { aide auditive } \\ & \text { micro-vibrateur } \\ \text { sonotone } & \text { verres de contact } \\ \text { verres de cornée } \\ \text { verres cornéens } \\ \text { lentilles de contact } \\ \text { lentilles cornéennes } \\ \text { software } \\ \text { logiciel } \\ \text { programmerie } \\ \end{array}$

Au moment de la création néologique, force nous est donc d'exclure le critère onomasiologique. Il nous faut donc retenir les critères suivants: l'intégration à la langue française (sur les plans phonologique, orthographique et syntaxique), l'absence de connotations et la valeur sociolinguistique.

\section{IMPORTANCE DE LA LEXIE COMPLEXE EN NÉOLOGIE FRANÇAISE}

Cherchons à voir maintenant comment le français réagit à la création néologique par la lexie complexe. Pour nous guider il convient de rappeler deux dépouillements d'importance qui, bien qu'ils ne soient pas récents, sont suffisamment fouillés pour nous être utiles et qu'il ne faudrait pas passer sous silence.

Le dépouillement extensif du journal Le Monde de 1955, relaté par Madame Lifetree-Majumdar, a donné les résultats suivants: sur 5049 néologismes, 1306 étaient formés au moyen de la composition, soit $25,87 \%$. Le néologisme

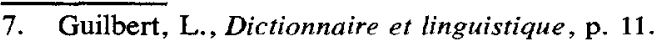


était défini comme une unité non présente dans les dictionnaires de langue française parus avant cette date. Force nous est donc d'accepter la conclusion de l'auteur: «la formation de mots composés est un procédé, ou plutôt un groupe de procédés, très fréquent en français moderne, surtout dans la langue écrite $\gg$.

Le groupe de procédés dont l'auteur a fait l'inventaire comprend :

-- l'apposition traditionnelle (amiante-ciment),

- l'apposition sans joncteur (camion-radio, sans de),

- l'apposition adjectivale (conférence fédérale-provinciale),

-- les syntagmes en voie de lexicalisation,

- de type substantif-de-substantif (bons d'épargne),

- de type préposition-substantif (hors-cadre),

- de type adverbe-composant (mal-nutrition),

- de type verbe-substantif (porte-skis),

- de type substantif-adjectif (presse filmée),

-- les dérivés de composés déjà existants (comédiens-français, académicien-

Goncourt),

- la composition

- dont un terme a une valeur suffixale (position-clé),

- sans valeur suffixale (cuir-velours),

- inversée (centre-Europe).

Si les procédés de formation de la lexie complexe sont nombreux et le recours à ce mode de création fréquent, il ne s'agit pas néanmoins des procédés prépondérants (du moins en langage journalistique de 1955). Ce procédé ne rend compte que de $25-26 \%$ des cas. On objectera sans doute qu'il s'agit là d'un dépouillement qui remonte au déluge (néologique). Faut-il alors répondre qu'il y a bien quelques Noé qui ont su sauver une barque pleine à craquer de créatures viables, et pas seulement des mille-pattes. La liste donnée par H. Joly ${ }^{9}$ ne compte que 10 lexies complexes sur 78 (soit $12,82 \%$ ).

Le rapport d'une lexie complexe sur quatre néologismes dégagé par Lifetree a bien souvent été dépassé. Un lexique sur l'énergie nucléaire ${ }^{10}$ donne 78 lexies complexes sur 92 entrées (soit une proportion de 84,78\%). Un comptage de 100 termes choisis au hasard dans les normes de l'ISO sur le vocabulaire informatique ${ }^{11}$ donne $78 \%^{12}$. Bien sûr, la lexie complexe s'avère un moyen adéquat pour spécifier des notions déjà connues :

8. Lifetree-Majumdar, J. J., Contribution à l'analyse des modes de composition nominale en français écrit contemporain, p. 65.

9. Joly, H. et al., «Französische Neologismen», in Lebende Sprachen, p. 163-167.

10. Kocher, H., "Petit glossaire des centrales nucléaires», in Lebende Sprachen, p. 159-163.

11. ISO 2382/IV; 1974.

12. À cet égard d'ailleurs, Robert Dubuc a déjà constaté le recours à la composition en néologie dans une proportion plus grande dans la langue technique $(61 \%)$ que dans la langue commune $(20 \%)$. Notons cependant que la lexie complexe est un des procédés de composition telle qu'entendue par Dubuc. 
- caractère

- jeu de caractères

- jeu partiel de caractères

- jeu partiel de caractères alphanumérique (?)

Mais est-ce là la façon la plus judicieuse?

Les résultats de l'étude menée par l'équipe d'André Phal ${ }^{13}$ doivent également être pris en considération, vu l'importance du corpus. Il faut rappeler que l'enquête menée par le CREDIF sur le VGOS (Vocabulaire Général d'Orientation Scientifique) avait pour but de dégager les critères servant au dépouillement mécanographique des lexies complexes trouvées dans les textes. La difficulté de définir ces critères d'une manière sûre était de taille, mais l'étude a permis de dégager un fait qu'il y a lieu de retenir: «[Il] semble bien que [le chiffre de quatre] soit un maximum: les groupes de mots constitués de plus de quatre composants à sens plein sont rares " ${ }^{14}$. Mais cette limite non plus n'est guère respectée. À titre d'exemple, on retrouve des expressions du type:

— presse à plier à plateau ajustable et à bigorne (Auger, P., p. 45);

- réacteur de puissance modéré par eau lourde et refroidi par gaz (Köcher, p. 160).

L'utilisation quasi exclusive (dans une proportion de 78 à $84.78 \%$ ) de la lexie complexe lors de la création néologique, et ses formes abusives (plus de quatre mots à sens plein), peuvent avoir de fâcheuses conséquences. Le recours à cette forme complexe fait passer subrepticement, mais sûrement, de l'activité purement dénominative de la création néologique à une activité essentiellement descriptive, quoique la ligne de démarcation entre les deux phénomènes soit difficile à établir. À preuve, la difficulté de définir certaines lexies complexes tout à fait explicites : comment définir la presse à plier à plateau ajustable et à bigorne sinon en étoffant, en fait presque inutilement : presse (qui sert) à plier (et dont le) plateau (peut être) ajusté et (ayant une) bigorne.

Une seconde conséquence, non moins gênante, se situe sur le plan syntaxique. Bien souvent, la lexie complexe fonctionne mal dans une phrase. Ce problème devient presque insoluble lorsqu'on est aux prises avec une phrase qui contient plus d'une lexie complexe, ce qui nous mène tout droit vers l'incongruité, sinon l'incompréhension pure et simple. L'ISO propose l'expression «jeu de caractères codés alphabétique" pour traduire alphabetic coded character set. Dès qu'on a deux jeux, la relation d'alphabétique à jeu se perd. Et qu'arrivera-t-il lorsque le jeu sera partiel et les caractères incomplets? Faudra-t-il parler de jeux partiels de caractères codés alphabétiques incomplets? Qui pourra penser qu'alphabétique se rapporte à jeu? On va donc à l'encontre d'un des critères précédemment cités par P. Auger et L.-J. Rousseau, en l'occurrence celui de l'intégration syntaxique.

13. Phal, A., Les groupes de mots, p. 45-60.

14. Ibid., p. 59 . 
Il n'y a pas que le niveau syntaxique qui pose un problème, mais aussi le niveau morphologique. La traduction de marketing en français est un cas classique. Mais si on rend marketing par mise en marché (ne discutons pas ici du fond, mais purement de la forme), comment traduire telemarketing? Mise en marché à distance par rapport à télécommercialisation, lequel des deux semble le plus apte à la dérivation?

\section{SOLUTIONS PROPOSÉES}

S'appuyant sur les raisons formulées précédemment, on pourrait légitimement chercher à rendre autrement que par le recours à la lexie complexe les nouvelles notions qui apparaissent. Du moins lorsque dans la langue de départ, cette notion est rendue par une lexie simple. Si nous revenons à nos deux problèmes de départ, voici quelques solutions qu'il ne faudrait pas rejeter du revers de la main : pour "visor», pourquoi pas visière, par extension de sens ${ }^{15}$; pour «multithreading», multifiliérisation. Ces solutions s'avéreraient peut-être les plus sages. En effet, si elles étaient appelées à évoluer, on rendrait plus facilement, par exemple :

$$
\begin{aligned}
& \text { multithreadable } \rightarrow \text { multifiliérisable } \\
& \text { autovisor } \rightarrow \text { autovisière }
\end{aligned}
$$

On pourrait taxer de témérité ces solutions, mais ne parlons-nous pas déjà de :

déprofessionnalisation, présidentialisation, praticable (dispositif de rangement et de manutention) ${ }^{16}$.

Chose certaine, les deux termes proposés respectent les critères d'intégration tant au plan orthographique qu'aux plans phonologique et syntaxique. De plus, ils semblent exempts de connotations. Ce ne sont peut-être pas là les meilleures solutions. Mais ce ne sont certes pas les pires!

RICHARD KROMP

\section{ANNEXE A}

Volet: Panneau (de menuiserie ou de métal) qui, placé à l'intérieur, sert à protéger le châssis d'une fenêtre, à intercepter la lumière. Par ext. Tout panneau, ou battant qui protège une baie. (1976) Techn. Ailette (d'une roue à aubes). Panneau articulé.

Visière : Pièce rigide qui protège les yeux et qui s'attache autour de la tête.

Déflecteur : Petit volet orientable d'une vitre de portière d'automobile, servant à aérer.

15. J'ai appris depuis que le $\mathrm{CN}$ a appelé visière la pièce rigide en forme d'arc et placée à la partie supérieure des feux de circulation ferroviaire. Visière semble donc commencer à prendre de nouveaux sens.

16. Joly, H., op. cit., p. 165. 


\section{ANNEXE B}

Thread. A group of beads

Bead. In a program a small module which performs a specific function. Beads are usually written and tested individually and then strung together and tested in groups sometimes known as threads. (Chandor A., The Penguin Dictionary of Computers, New York, Penguin Books, 1977)

Filière: (Système IBM 8100) Enchaînement des différentes tâches utilisateur et système qui concourent à la réalisation d'un travail. (IBM France, Terminologie du traitement de l'information, Paris, IBM France, 1980)

Multithread: Used on a program which can have more than one logical path through it being executed simultaneously. (Sippl, C. J., Data Communications Dictionary, New York, Van Nostrand Reinhold, 1976)

\section{BIBLIOGRAPHIE SOMMAIRE}

AUGER, P. et L.-J. ROUSSEAU, Études, recherches et documentation. Méthodologie de la recherche terminologique, Québec, Éditeur du Québec, 1978, 80 p.

BOUTIN-QUESNEL, R. et al, Études, recherches et documentation. Vocabulaire systématique de la terminologie, Québec, Éditeur du Québec, 1979, 87 p.

DUBUC, R., Manuel pratique de terminologie, Montréal, Linguatech, 1978, 102 p.

DUBUC, R., «A l'écoute de la langue vivante», Meta, vol. 25, nº 2, juin 1980, p. 249-253.

GUILBERT, L., «Dictionnaires et linguistique : essai de typologie des dictionnaires monolingues français contemporains », Langue française, $\mathrm{n}^{\circ} 2,1969$, p. 4-29.

GUILBERT, L., "La spécificité du terme scientifique et technique », Langue française, vol. 17, février 1973, p. 5-17.

ISO 2382/IV, 1974.

JOLY, H., Y. LANCEL et C. PLONK, «Franzosische Neologismen», Lebende Sprachen, ${ }^{\circ}$ 4, décembre 1976, p. 159-163.

KÖCHER, H., «Petit glossaire des centrales nucléaires », Lebende Sprachen, n 4, décembre 1977, p. 159-163.

PHAL, A., «Les groupes de mots et les problèmes qu'ils posent dans la préédition de textes scientifiques destinés à l'analyse mécanographique ", Cahiers de lexicologie, $n^{\circ} 4$, p. 45-60.

STEINER, R. J., «Neologisms and Scientific Words in Bilingual Lexicography: Ten Problems", Lebende Sprachen, $\mathrm{n}^{\circ} 4$, décembre 1976, p. 145-150. 